\title{
Fabrication of DNA nanotubes using origami-based nanostructures with sticky ends
}

\author{
Mohammad Mousavi-Khattat • Adele Rafati • \\ Pooria Gill
}

Received: 30 October 2014/ Accepted: 16 January 2015/Published online: 5 February 2015

(C) The Author(s) 2015. This article is published with open access at Springerlink.com

\begin{abstract}
Described here is a simplified method for fabrication of DNA nanotubes using a minimum numbers of staple oligomers for DNA origami. For this purpose, the cylindrical nanotemplates with two sticky ends have been designed using caDNAno software. Then, the nanostructures were shaped in an optimized experimental condition via an origami-based self-assembly reaction. Finally, the produced nanostructures were joined together through their sticky ends using a ligase enzyme. Transmission electron microscope confirmed fabrication of these elongated nanotubes. In addition, high-resolution microscopy of DNA nanotubes by scanning tunnelling microscope indicated efficient attachments of the primarily DNA nanostructures via their sticky ends. The results demonstrated that a ligase treatment of cylindrical DNA nanostructures with the sticky ends made DNA nanotubes with standard shapes using minimum numbers of staples.
\end{abstract}

Keywords DNA nanotube $\cdot$ T4 ligase $\cdot$ Sticky ends . TEM $\cdot$ STM

M. Mousavi-Khattat · A. Rafati · P. Gill

Department of Medical Biotechnology, Faculty of Advanced

Medical Technologies, Golestan University of Medical Sciences,

Gorgan, Iran

P. Gill

Nanomedicine Group, Research Center for Immunogenetics, Mazandaran University of Medical Sciences, Sari, Iran

P. Gill $(\square)$

Department of Physio Pharmacology and NanoBioMedicine, Faculty of Medicine, Mazandaran University of Medical Sciences, Sari, Iran

e-mail: pooriagill@yahoo.com; p.gill@mazums.ac.ir

\section{Introduction}

DNA nanotechnology uses DNA as a building block to create nano-scale structures which acts as a candidate in multidisciplinary applications such as fabrications of nanodevices, nanoarrays, nanodiagnostics, nanotherapeutics, and the other demanding areas [1-4]. Among DNA nanostructures, DNA nanotubes have particularly potentials for functions due to high aspect ratio [5], controllability in fabrications [6, 7], and some capabilities for applications as nanocarriers [8], nanoarrays [9], nanowires [10], nanotools for structural analysis [11], and nanoanalytes for controlling some fabrication processes [12].

DNA origami was introduced as a new growth mechanism for DNA assembly for fabrication of various shapes of DNA nanostructures. In this technique, a long single-stranded DNA templated the final structure, so-called DNA scaffold [13]. DNA origami gives excellent control over the size and shape of objects $[14,15]$. However, this level of structural complexity requires hundreds of staple strands with different sequence as well as long annealing times [16]. In other words, obtaining nanotubes with high aspect ratio need more quantities of staple oligonucleotides within a DNA origami procedure; however, more staples in fabrication made more costs in practice. Hence, we aimed here to introduce a simple procedure for fabrication of DNA nanotubes via an origamibased technique assisted with a ligase reaction. This fabrication process provides DNA nanotube with minimum numbers of the staples within a self-assembly reaction.

In this study, computational modelling of DNA nanotubes performed using caDNAno software [17] that superimposed a cylindrical scaffold with staples as essentials in a self-assembly process. The nanotemplates had sticky ends for jointing together; the nanotubes were made with high aspect ratio (Fig. 1). 
Fig. 1 Schematic fabrication of DNA nanotubes designed by caDNAno. a Three-dimensional graphical view of DNA nanotemplates with sticky ends; b DNA nanotemplates jointed together by ligase treatment; c the positions of sticky ends in ligation for shaping DNA nanotubes with high aspect ratio (a)

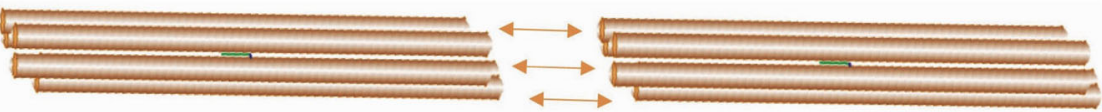

(b)

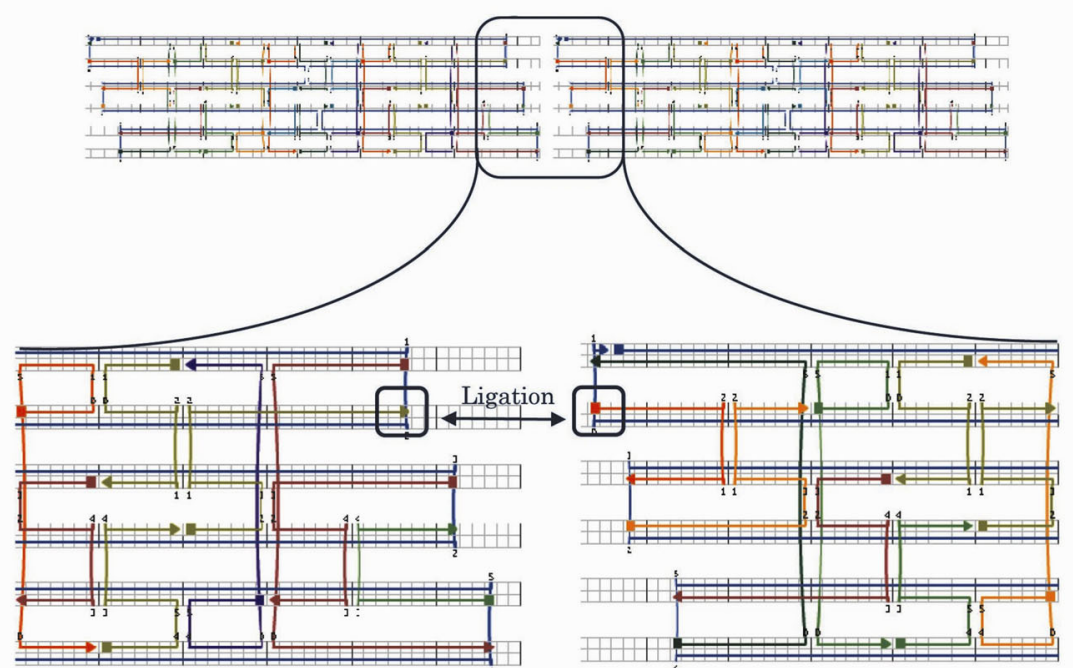

The cylindrical nanostructures were constructed using $\mathrm{M}_{13} \mathrm{mp}_{18}$ DNA as scaffold and staples with specific sequences. The nanotemplates jointed together to form DNA nanotubes via a ligase reaction.

\section{Materials and methods}

Chemicals and instruments

Thermal condition for self-assembly in origami reaction was set using thermocycler CA 1000 (Bio-Rad, USA). Gel electrophoresis experiments were performed using electrophoresis mini set from Bio-Rad (USA). The micrographs were obtained by NAMA-STM SS-3 (Nano System Pars Corporation, Natsyco, Iran) and TEM (Philips EM028, USA). Highly ordered pyrolytic graphite (HOPG) was prepared from Nano System Pars Corporation. $\mathrm{M}_{13} \mathrm{mp}_{18}$ phage genome was purchased from New England Biolabs (USA). $\mathrm{T}_{4}$ DNA ligase was provided by Thermo (USA) and the desired single strand oligonucleotides were synthesised by Sigma. SYBRGold gel staining solution was purchased from Molecular Probes Inc. (Oregon, USA). Quantum Prep ${ }^{\mathrm{TM}}$ Freeze 'N Squeeze DNA Gel Extraction Spin Columns were from Bio-Rad, USA.

Computational modelling of nanotube-based templates with sticky ends

Recently caDNAno is an attractive software for scientists to design variable origami shapes. It commonly applies two inputs, including a lengthy scaffold and a group of crossing staples. M13mp18 DNA was used as DNA scaffold and staples were designed based on their complementarities with special sites of the scaffold sequences for shaping sticky-ended nanostructures.

Self-assembly of nanotube-based templates

Assembly of DNA origami nanotubes was accomplished by mixing $10 \mathrm{nM} \mathrm{M}_{13} \mathrm{mp}_{18}$ DNA with $100 \mathrm{nM}$ of each staple oligonucleotide. This self-assembly mixture contained TAE- $\mathrm{Mg}^{2+}(40 \mathrm{mM}$ Tris, $20 \mathrm{mM}$ acetic acid, $2 \mathrm{mM}$ EDTA and $12.5 \mathrm{mM}$ magnesium acetate, $\mathrm{pH}$ 8.0). Thermal annealing program was applied via a hybridization program from 90 to $25^{\circ} \mathrm{C}$ with $1{ }^{\circ} \mathrm{C} / \mathrm{min}$ ramping rate.

Ligase treatment of the nanotube templates

Ligation was the next step of the fabrication method that resulted longitudinal binding of primarily short DNA nanotubes together. For this purpose, $10 \mu \mathrm{l}$ origami product, $2 \mu \mathrm{l} 10 \times \mathrm{T}_{4}$ DNA ligation buffer, $2 \mu \mathrm{l} 50 \%$ PEG 4,000 solution, $5 \mathrm{U} \mathrm{T}_{4}$ DNA ligase, and nuclease-free water were mixed up to $20 \mu \mathrm{l}$ in a total volume. The mixture was incubated for $1 \mathrm{~h}$ at $22{ }^{\circ} \mathrm{C}$ according to the manufacturer's instruction.

Gel electrophoresis

The origami product was loaded into the gel electrophoresis wells after completing the folding phase immediately. 
Fig. 2 The caDNAno decoration of a DNA nanotubebased template with two sticky ends. The scaffold DNA was differentiated from the staples with blue colour. Not all the 24 staples were hybridized to the scaffold DNA into doublestranded DNAs. Many of the staples enclosed to the scaffold into crossing-over strands and holiday junctions

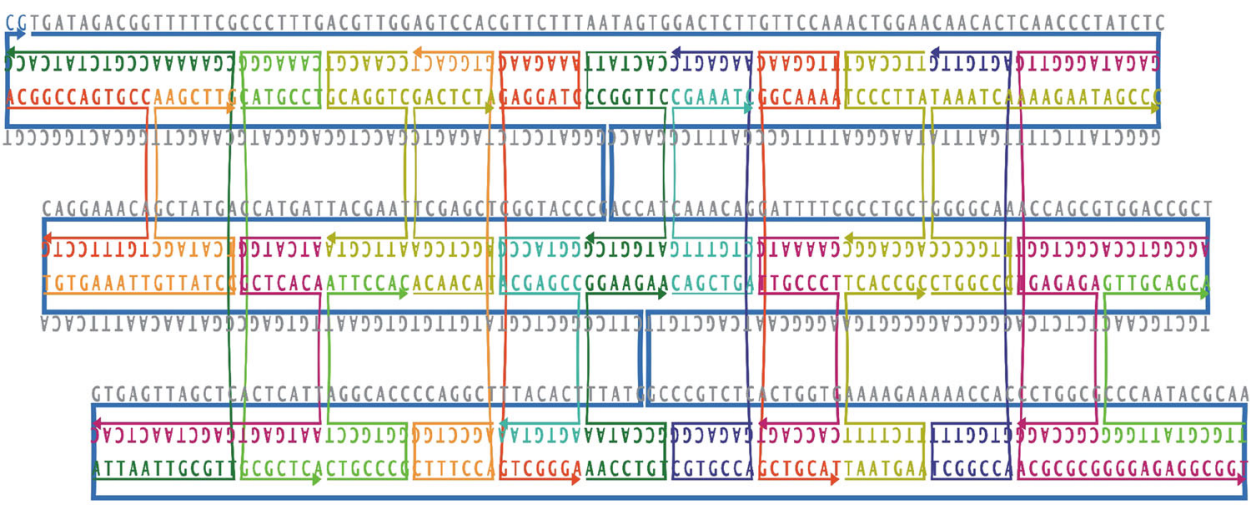

The experiment was performed in $1 \%$ agarose gel at TAE buffer with a voltage of $100 \mathrm{~V}$ applied in $25 \mathrm{~min}$ by power supply. Gel staining was performed by SYBRGold working solution. In addition, the ligation product was run in the same experimental condition for the gel electrophoresis. After electrophoresis, DNA nanotubes were extracted from the gels using Quantum Prep ${ }^{\mathrm{TM}}$ Freeze ' $N$ Squeeze DNA Gel Extraction Spin Columns according to the manual instruction.

Transmission electron microscopy of DNA nanotubes

Transmission electron microscope was used to determine the size and morphology of DNA nanotubes. For this purpose, the samples were immobilized by syringe spraying on agar scientific holey carbon film with 300 mesh $\mathrm{Cu}_{(50)}$.

\section{Characterization of DNA nanotubes by STM}

The ligation product-contained DNA nanotubes were diluted $10^{3}$ folds in TAE- $\mathrm{Mg}^{2+}$ buffer ( $\mathrm{pH}$ 8). Then, $5 \mu \mathrm{l}$ diluted sample was immobilized on the HOPG by drying for $3 \mathrm{~h}$ at room temperature [18]. The samples were imaged using topographic mode with STM, with $0.1 \mathrm{nA}$ current set point and $0.2 \mathrm{~V}$ sample bias through a platinum iridium tip. Rough data were first processed using line adjust, plain adjust and average filters of the NAMA-STM Nanoanalyzer software. Then, the colouring process was tested on the obtained micrographs for different levels [19].

\section{Results}

DNA nanotube templates with sticky ends

The modelled nanotube obtained from caDNAno software was demonstrated in Fig. 2. The model consisted of a cylindrical scaffold assembled with the staples from the oligonucleotides. The scaffold has been formed from six single-stranded DNA with the M13mp18 nucleotide contents that were shown with blue colour. In addition, sequences of the staples were determined as those applied on the scaffold and eventually 24 staples (Table 1) were determined with the variable lengths.

The 24 staples were indicated with different colours in the computational model. The computerized nanotube contained two sticky ends that those could be jointed to their complements for shaping DNA nanotubes via a ligase reaction.

Table 1 Staple oligomers for self-assembly of DNA nanotemplates

\begin{tabular}{ll}
\hline $\begin{array}{l}\text { Oligomer } \\
\text { name }\end{array}$ & Sequence $\left(5^{\prime}-3^{\prime}\right)$ \\
\hline $\mathrm{S}_{1}$ & CCAACGTGCAGGTCATTCGTA \\
$\mathrm{S}_{2}$ & CACTATTCCGGTTCATGGTCG \\
$\mathrm{S}_{3}$ & TTCCAGTTCCCTTAAGCAGGC \\
$\mathrm{S}_{4}$ & GAGATAGGGTTGACGCGCGGGGAGAGGCGGT \\
$\mathrm{S}_{5}$ & ACGGCCAGTGCCTGTTTCCTG \\
$\mathrm{S}_{6}$ & CATGCCTCAAAGGGGCGCTCA \\
$\mathrm{S}_{7}$ & GAGGATCAAAGAACGTCGGGA \\
$\mathrm{S}_{8}$ & GGCAAAATTGGAACGCTGCAT \\
$\mathrm{S}_{9}$ & ATCATGGGCTCACAAATGAGTGAGCTAACTCAC \\
$\mathrm{S}_{10}$ & GGTACCGACGAGCCAGTGTAA \\
$\mathrm{S}_{11}$ & GAAAATCTTGCCCTCACCAGT \\
$\mathrm{S}_{12}$ & AGCGGTCCACGCTGGTTGAGAGACGCCAGG \\
$\mathrm{S}_{13}$ & TGTGAAATTGTTATCCTCATAGCAAGCTTG \\
$\mathrm{S}_{14}$ & ACAACATAGCTCGAGACTCTA \\
$\mathrm{S}_{15}$ & CAGCTGACTGTTTGCGAAATC \\
$\mathrm{S}_{16}$ & CTGGCCCTTGCCCCTAAATCAAAAGAATAGCCC \\
$\mathrm{S}_{17}$ & AGCCTGGCTTTCCAGTGGACT \\
$\mathrm{S}_{18}$ & GAGACGGCGTGCCAAAGAGTC \\
$\mathrm{S}_{19}$ & GTGGTTTTCGGCCAAGTGTTG \\
$\mathrm{S}_{20}$ & TTGCGTATTGGGGTTGCAGCA \\
$\mathrm{S}_{21}$ & ATTAATTGCGTTCGAAAAACCGTCTATCACG \\
$\mathrm{S}_{22}$ & CTGCCCGGGTGCCTATTCCAC \\
$\mathrm{S}_{23}$ & AACCTGTGCCATAAGGAAGAA \\
$\mathrm{S}_{24}$ & TAATGAATTCTTTTCACCGC \\
\hline & \\
\hline
\end{tabular}




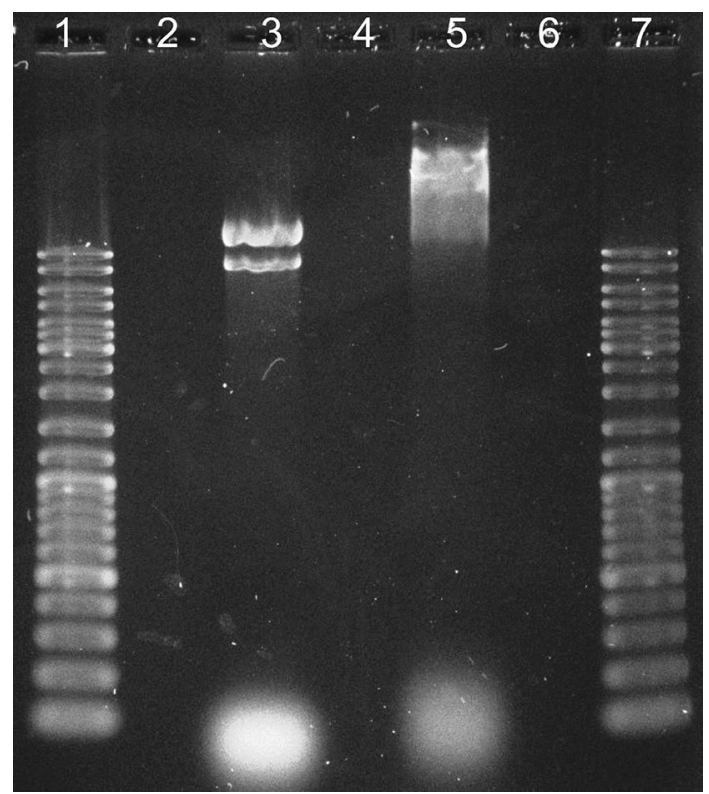

Fig. 3 Electrophoresis behaviours of DNA nanotubes before and after $\mathrm{T}_{4}$-ligase treatment. Lanes 1 and 7, DNA ladder 100-10,000 bp (Thermo, USA); lane 3, DNA nanotemplates fabricated via origami self-assembly; lane 5, elongated DNA nanotubes after ligation step

\section{Electrophoresis behaviours of DNA nanotubes}

Gel electrophoresis behaviours of DNA nanotubes after ligase treatment were checked in comparison to the DNA nanotemplates obtained from origami-based assembly (Fig. 3). The results demonstrated that the ligated DNA nanotubes had higher molecular weight than those nanotemplates before ligase treatment. The fact confirmed that the ligase treatment has occurred successfully and it made the nanotubes shape via ligation of the primarily nanotemplates together, periodically.

\section{TEM micrographs}

TEM micrograph of DNA nanotubes has been shown in Fig. 4. The nanotubes were in filamentous shapes. The nanotubes were in micron sizes at lengths and their morphologies confirmed their fabrication efficiently.

\section{STM micrographs of DNA nanotubes}

Figure 5a indicated STM micrograph of DNA nanotubes with zoom in their structures. The 2D micrograph of the nanotubes demonstrated the highly ordered self-assembly and self-organization of nanotemplates, clearly; moreover, the sticky ends of the primary nanotemplates could be jointed successfully together among the elongated DNA nanotubes. Size diagram of the micrograph (Fig. 5b) confirmed that the nanotubes had a similar size in their ends

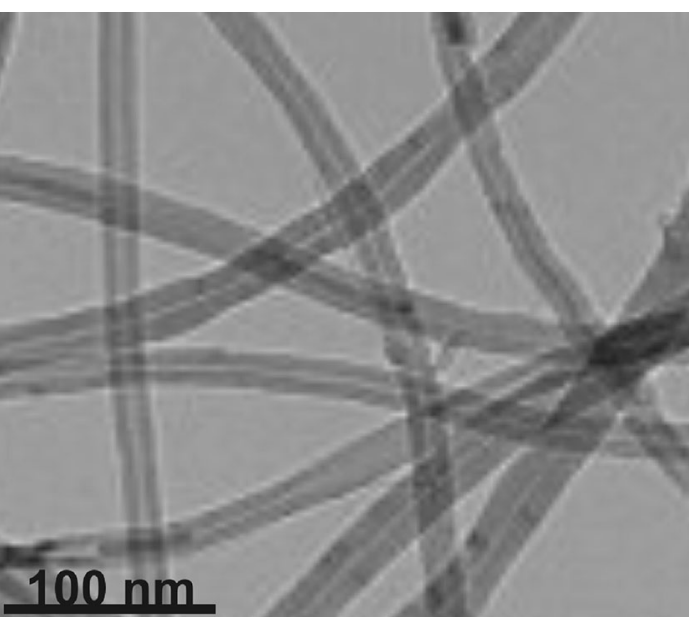

Fig. 4 TEM micrograph of DNA nanotubes. The nanotubes have micron lengths with nano-scale widths. It seems the nanotubes were not seen in monolayer; hence, some variations were seen in nanotubes. Transmission electron microscopy was done using Philips EM028 transmission electron microscope

measured nearly $10-15 \mathrm{~nm}$ in $Z$-axis and $35-40 \mathrm{~nm}$ in $L$ axis, respectively.

\section{Discussion}

DNA as nanomaterial has flexibility to make programmable shapes resulting via base-pairing rules in DNA assembly phenomenon [20-22]. There are two ways to form DNA nanostructures; first by making a large structure from specialized oligonucleotides known as a bottom-up approach in fabrication [23]; second by shaping a large molecule that will create a programmable structure that needs to be minimized as a top-down approach in fabrication [24]. According to the ways in DNA nanofabrication, various shapes of nano-scaled DNA structures have been created in octahedron [25], cubic [26], icosahedron [27], and tube [28-31] forms.

Nanotubes are promising nanomaterials not only at the nano-scale applications, but also they can play a functional role at visual scales, according to their components and designs; however, all of these applications are taking advantages of nanotubes properties, such as their high aspect ratio and size variation [1-4]. A beneficial material creating these nanostructures may be DNA, with the highest specificity in the assembly and the most simplicity in their fabrications and organizations. Moreover, various softwares have been employed for modelling different structures of DNAs that those would be easier for use in DNA nanotubes [24, 31].

In practice, thermal self-assembly and the isothermal assembly using denaturing agents [32] were two ways for 

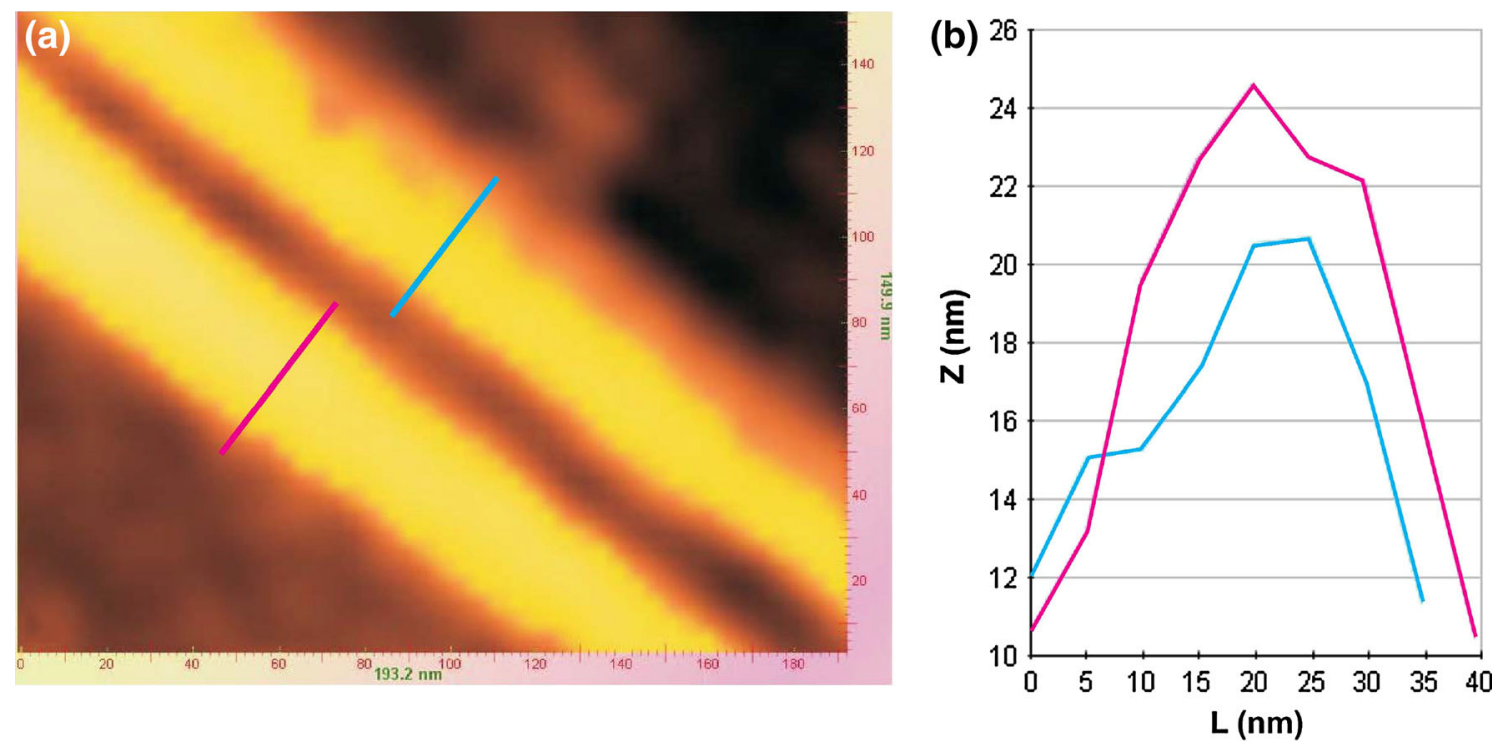

Fig. 5 High resolution of STM micrograph from two DNA nanotubes on HOPG surface. a 2D image of two DNA nanotubes with more details of their structures. b Size diagram of two DNA nanotubes measured nearly $10-15 \mathrm{~nm}$ in height $(Z)$ with one jump on diagram; and 35-40 nm in length $(L)$ of the jump, respectively. The

creating DNA nanostructures; however, many staple oligonucleotides would be needed in the self-assembly of the nanotubes and lead to more costs for obtaining the nanotubes with more lengths. On the other hand, the obtained products would be labile particularly for imaging by electron or scanning probe microscopies [28, 33].

Here, we introduced a fabrication way accomplished with the origami technique and ligation for providing DNA nanotubes with standard dimensions. The diameter of DNA nanotubes could control according to the numbers of circles selected in caDNAno software. In this study, six graphical circles were selected for shaping cylindrical nanotemplates of DNA nanotubes. Moreover, it seems more length of DNA nanotube shaped via selecting more lengths of cylindrical nanotemplates designed by caDNAno software. This procedure made beneficial nanofabrication of nanotubes by employing minimum staples. In addition, this procedure for fabrication of DNA nanotubes would provide a suitable condition for a high-resolution imaging by STM; however, to our knowledge, STM characterization of DNA nanotubes has not been reported, previously.

The nanotubes were resulted via periodic ligation of the primarily self-assembled nanotemplates; however, the gel electrophoresis experiments also confirmed the different weights between the primarily templates of DNA nanotubes and the ligated forms.

Ligation could make elongation of the primarily nanotubes via connecting the sticky ends and their growth in length; hence, $\mathrm{T}_{4}$ ligase could also stabilized DNA nanotubes; make them more robust for imaging by STM. image has been obtained by NAMA-STM SS-3L1 (Nanotech System Corporation, Natsyco, Iran). Current set point and sample bias voltage were set at $0.1 \mathrm{nA}$ and $0.2 \mathrm{~V}$, respectively. Rough data were filtered by line, plain adjusts, and average filters of NAMA-STM SS3-L1 Nanoanalyzer software

Alternatively, similar report on the tile-based lattices and their ligation for more fully ligatable DNA lattices has been published, previously [28]; however, the construction of DNA nanotubes in that report has not been shaped directly via caDNAno-based origami self-assembly described here.

\section{Conclusion}

Because of the high aspect ratio of DNA nanotubes, these nanoarchitectures have been candidate for various applications in biology, nanotechnology, and medicine, i.e. nanomedicine. DNA nanotubes have also been employed as nanowires loaded with various nanoparticle types and suggested them as electrical conductors [34, 35]. DNA nanotubes could also be applied in drug delivery systems because of their high capacity for carrying more cargoes [5, 8]. The nanotubes could also be substituted with carbon nanotubes due to their compatibilities with the life systems [36]. Therefore, design and development of a cost benefit procedure for simply fabrication of DNA nanotubes would be essential. It seems fabrication of DNA nanotubes via ligation of DNA nanotemplates with designed sticky ends could be a simplified procedure for providing stabilized DNA nanotubes with the minimum staples when assisted with a ligase treatment. This study also demonstrated the role of STM in high-resolution imaging of DNA nanotubes in air condition with no use of the vacuumed chamber commonly required in electron microscopes. 
Author contribution $\mathrm{P}$. Gill conceived and designed the experiments. S-M. Mousavi-Khattat and A. Rafati equally performed the experiments. P. Gill analysed the data. P. Gill contributed reagents/materials/analysis tools. S-M. Mousavi-Khattat and P. Gill wrote the paper.

Acknowledgments This study was supported by Vice Chancellor of Research and Technology, Golestan University of Medical Sciences Grant no. 352438. Also, Iran Nanotechnology Initiative (INI) supported this project partially. The authors thank Batool Yousofi for her collaboration with this study.

Open Access This article is distributed under the terms of the Creative Commons Attribution License which permits any use, distribution, and reproduction in any medium, provided the original author(s) and the source are credited.

\section{References}

1. Seeman, N.C.: Nucleic acid junctions and lattices. J. Theor. Biol. 99, 237-247 (1982)

2. Dietz, H., Douglas, S.M., Shih, W.M.: Folding DNA into twisted and curved nanoscale shapes. Science 325, 725-730 (2009)

3. Winfree, E., Liu, F., Wenzler, L.A., Seeman, N.C.: Design and self-assembly of two-dimensional DNA crystals. Nature 394, 539-544 (1998)

4. Seeman, N.C.: DNA in a material world. Nature 421, 427-431 (2003)

5. Lo, P.K., Karam, P., Aldaye, F.A., McLaughlin, C.K., Hamblin, G.D., Cosa, G., Sleiman, H.F.: Loading and selective release of cargo in DNA nanotubes with longitudinal variation. Nat. Chem. 2, 319-328 (2010)

6. Sharma, J., Chhabra, R., Cheng, A., Brownell, J., Liu, Y., Yan, H.: Control of self-assembly of DNA tubules through integration of gold nanoparticles. Science 323, 112-116 (2009)

7. Wang, R., Liu, W., Seeman, N.C.: Prototyping nanorod control: a DNA double helix sheathed within a DNA six-helix bundle. Chem. Biol. 16, 862-867 (2009)

8. Ko, S.H., Liu, H., Chen, Y., Mao, C.: DNA nanotubes as combinatorial vehicles for cellular delivery. Biomacromolecules $\mathbf{9}$, 3039-3043 (2008)

9. Bui, H., Onodera, C., Kidwell, C., Tan, Y., Graugnard, E., Kuang, W., Lee, J., Knowlton, W.B., Yurke, B., Hughes, W.L.: Programmable periodicity of quantum dot arrays with DNA origami nanotubes. Nano Lett. 10, 3367-3372 (2010)

10. Liu, D., Park, S.H., Reif, J.H., LaBean, T.H.: DNA nanotubes self-assembled from triple-crossover tiles as templates for conductive nanowires. Proc. Nat. Acad. Sci. USA 101, 717-722 (2004)

11. Douglas, S.M., Chou, J.J., Shih, W.M.: DNA-nanotube-induced alignment of membrane proteins for NMR structure determination. Proc. Nat. Acad. Sci. USA 104, 6644-6648 (2007)

12. Wang, T., Schiffels, D., Martinez-Cuesta, S., Kuchnir-Fygenson, D., Seeman, N.C.: Design and characterization of 1D nanotubes and 2D periodic arrays self-assembled from DNA multi-helix bundles. J. Am. Chem. Soc. 134, 1606-1616 (2012)

13. Rothemund, P.W.: Folding DNA to create nanoscale shapes and patterns. Nature 440, 297-302 (2006)

14. Douglas, S.M., Dietz, H., Liedl, T., Hogberg, B., Graf, F., Shih, W.M.: Self assembly of DNA into nanoscale three dimensional shapes. Nature 459, 414-418 (2009)
15. Han, D., Pal, S., Nangreave, J., Deng, Z., Liu, Y., Yan, H.: DNA origami with complex curvatures in three-dimensional space. Science 332, 342-346 (2011)

16. Hamblin, G.D., Hariri, A.A., Carneiro, K.M.M., Lau, K.L., Cosa, G., Sleiman, H.F.: Simple design for DNA nanotubes from a minimal set of unmodified strands: rapid, room-temperature assembly and readily tunable structure. ACS Nano 7, 3022-3028 (2013)

17. Douglas, S.M., Marblestone, A.H., Teerapittayanon, S., Vazquez, A., Church, G.M., Shih, W.M.: Rapid prototyping of 3D DNAorigami shapes with caDNAno. Nucleic Acids Res. 37, 5001-5006 (2009)

18. Gill, P., Ranjbar, B., Saber, R.: Scanning tunneling microscopy of cauliflower-like DNA nanostructures synthesized by loop-mediated isothermal amplification. IET Nanobiotechnol. 5, 8-13 (2011)

19. Saber, R., Sarkar, S., Gill, P., Nazari, B., Faridani, F.: High resolution imaging of $\mathrm{IgG}$ and $\mathrm{IgM}$ molecules by scanning tunneling microscopy in air condition. Sci. Iran 18, 1643-1646 (2011)

20. Watson, J.D., Crick, F.H.C.: Molecular structure of nucleic acids: a structure for deoxyribose nucleic acid. Nature 171, 737-738 (1953)

21. He, Y., Ye, T., Su, M., Zhang, C., Ribbe, A.E., Jiang, W., Mao, C.: Hierarchical self-assembly of DNA into symmetric supramolecular polyhedral. Nature 452, 198-201 (2008)

22. Sun, X., Ko, S.H., Zhang, C., Ribbe, A.E., Mao, C.: Surfacemediated DNA self-assembly. J. Am. Chem. Soc. 131, 13248-13249 (2009)

23. Sherman, W.B., Seeman, N.C.: A precisely controlled DNA biped walking device. Nano Lett. 4, 1203-1207 (2004)

24. Lin, C., Ke, Y., Liu, Y., Mertig, M., Gu, J., Yan, H.: Functional DNA nanotube arrays: bottom-up meets top-down. Angew. Chem. 46, 6089-6092 (2007)

25. Shih, W.M., Quispe, J.D., Joyce, G.F.: A 1.7-kilobase singlestranded DNA that folds into a nanoscale octahedron. Nature 427, 618-621 (2004)

26. Andersen, E.S., Dong, M., Nielsen, M.M., Jahn, K., Subramani, R., Mamdouh, W., Golas, M.M., Sander, B., Stark, H., Oliveira, C.L.P., Pedersen, J.S., Birkedal, V., Besenbacher, F., Gothelf, K.V., Kjems, J.: Self-assembly of a nanoscale DNA box with a controllable lid. Nature 459, 73-76 (2000)

27. Zhang, C., Su, M., He, Y., Zhao, X., Fang, P.A., Ribbe, A.E., Jiang, W., Mao, C.: Conformational flexibility facilitates selfassembly of complex DNA nanostructures. Proc. Nat. Acad. Sci. USA 105, 10665-10669 (2008)

28. Neill, P.O., Rothemund, P.W.K., Kumar, A., Fygenson, D.K.: Sturdier DNA nanotubes via ligation. Nano Lett. 6, 1379-1383 (2006)

29. Endo, M., Seeman, N.C., Majima, T.: DNA tube structures controlled by a four-way-branched DNA connector. Angew. Chem. 44, 6074-6077 (2005)

30. Qian, P., Seo, S., Kim, J., Kim, S., Lim, B.S., Liu, W.K., Kim, B.J., Labean, T.H., Park, S.H., Kim, M.K.: DNA nanotube formation based on normal mode analysis. Nanotechnology 23, 105704 (2012)

31. Yin, P., Hariadi, R.F., Sahu, S., Choi, H.M.T., Sung, H.P., LaBean, T.H., Reif, J.H.: Programming DNA tube circumferences. Science 321, 824-826 (2008)

32. Jungmann, R., Liedl, T., Sobey, T.L., Shih, W., Simmel, F.C.: Isothermal assembly of DNA origami structures using denaturing agents. J. Am. Chem. Soc. 130, 10062-10063 (2008)

33. Hamblin, G.D., Carneiro, K.M.M., Fakhoury, J.F., Bujold, K.E., Sleiman, H.F.: Rolling circle amplification-templated DNA nanotubes show increased stability and cell penetration ability. J. Am. Chem. Soc. 134, 2888-2891 (2012) 
34. Monson, C.F., Woolley, A.T.: DNA-templated construction of copper nanowires. Nano Lett. 3, 359-363 (2003)

35. Ford, W.E., Harnack, O., Yasuda, A., Wessels, J.M.: Platinated DNA as precursors to templated chains of metal nanoparticles. Adv. Mater. 13, 1793-1797 (2001)
36. Bianco, A., Kostarelos, K., Prato, M.: Applications of carbon nanotubes in drug delivery. Cur. Opin. Chem. Biol. 9, 674-679 (2005) 